\title{
Aprendizaje de Sistemas Matemáticos de Símbolos en Álgebra Lineal y Cálculo
}

\author{
Mathematical Symbol Systems Learning in Linear Algebra and Calculus
}

\author{
Eduardo Mario Lacués Apud*
}

\begin{abstract}
Resumen
Este reporte presenta los efectos sobre los aprendizajes de un diseño didáctico, con la finalidad de enseñar Sistemas Matemáticos de Símbolos (SMS) en un primer curso universitario de Álgebra Lineal, basado en la retroalimentación a los estudiantes a partir de su desempeño en tareas de resolución de ejercicios. Se reporta complementariamente el estudio de la incidencia de esta intervención en los desempeños de los alumnos en un curso de Cálculo. Se utilizó un diseño experimental con un grupo experimental (GE) y uno de control (GC), con un test inicial y otro final para evaluar desempeños. Se clasificó a los estudiantes mediante el test inicial en dos categorías (calificación alta- calificación baja) y se estableció que los de calificación alta de GE consiguieron desempeños en el test final significativamente mayores que los correspondientes de GC (se utilizó una prueba de Mann-Whitney). No se detectaron diferencias entre los subgrupos de estudiantes de calificación baja. Los integrantes de GE tuvieron desempeño significativamente mayor que los de GC en la tarea de Cálculo (se utilizó un Student's t-text). Estos resultados permiten utilizar este diseño en grupos de estudiantes con alta calificación inicial, y desafía a buscar estrategias alternativas para enseñar a los de menor calificación.
\end{abstract}

Palabras-Clave: Aprendizaje. Sistemas Matemáticos de Símbolos (SMS). Álgebra Lineal. Cálculo.

\begin{abstract}
This report presents the effects on learning of an instructional design for teaching the role of Mathematical Symbol Systems (MSS) in a first year college Linear Algebra course. based on feedback to students regarding their performance in tasks consisting of solving exercises. To complement it, a study is also described of the impact of this intervention on the performance of students in a calculus course. An experimental design was used with an experimental group (EG) and a control (CG), with initial and final tests to evaluate performance. Students were classified based on the initial test into two categories (high rating - low rating), and it was established that the high rating students of the EG scored significantly higher in the final test performance than those of the CG (Mann-Whitney test was used). No differences were detected between subgroups of low rating groups. EG members had significantly higher scores than those of CG in Calculus's tasks (we used a Student's t test). These results suggest the design can be used with groups of students with high initial rating, while posing the problem of finding alternative strategies to teach students with low initial rating.
\end{abstract}

Keywords: Learning. Mathematical Symbol Systems (MSS). Linear Algebra. Calculus.

\footnotetext{
${ }^{*}$ Magister en Educación por la Universidad Católica del Uruguay (UCU). Profesor Titular de tiempo completo en el Departamento de Matemática de la Facultad de Ingeniería y Tecnologías (FIT) de la Universidad Católica del Uruguay (UCU), Montevideo, Uruguay. Dirección Postal: Avenida 8 de Octubre, 2738, Tres Cruces, CP 11600, Montevideo, Montevideo, Uruguay. E-mail: elacues@ucu.edu.uy.
} 


\section{Relevancia de los SMS y necesidad de su enseñanza}

Los Sistemas Matemáticos de Símbolos (SMS) son una clase particular de sistemas externos de representación.

Hablar de sistemas externos de representación refiere a una combinación consensuada de signos que sirve para describir alguna entidad, relacionando algunas características de lo representado a través de algunas particularidades en la configuración simbólica que se está usando como representante.

Al mencionar los procesos de adquisición de sistemas de representación externa, Martí y Pozo (2000) dan cuatro aspectos de los sistemas externos de representación:

i) Existen en forma independiente de su creador.

ii) Poseen permanencia en el tiempo, al ser marcas hechas sobre algún soporte material.

iii) Están desplegados en el espacio, no en el tiempo, a diferencia del lenguaje hablado o gestual.

iv) Constituyen estructuras organizadas consensuadamente.

Estas características explican alguna de sus funciones, como la comunicativa; por otro lado, permiten anticipar algunas de las dificultades para su apropiación por parte de los aprendices, ligadas al carácter hasta cierto punto arbitrario de la organización de cada sistema de representación externa. Sobre este tema se volverá más adelante.

Según Palmer (1978) esta noción de representación externa implica la aceptación de la existencia de dos mundos, uno representado y otro representante, que se relacionan entre sí; siguiendo con su exposición, para disponer de un sistema de representación es necesario atender a los cinco aspectos siguientes:

a) Cuál es el mundo representado.

b) Cuál es el mundo representante.

c) Cuáles aspectos del mundo representante constituyen el modelo.

d) Cuáles aspectos del mundo representado van a ser modelados.

e) Cuáles son las correspondencias entre los dos mundos.

En la línea de esta noción, Kaput (1987) da una definición de lo que entiende por SMS: comienza por definir un esquema de símbolos como una colección realizable concretamente de caracteres, junto con reglas más o menos explícitas para identificarlos y 
combinarlos; una terna formada por un esquema de símbolos, un campo de referencia y una ley que establece una correspondencia entre los dos anteriores es un SMS.

Como una peculiaridad distintiva de los SMS, remarca que son sistemas de símbolos cuyo mundo representado es, a su vez, un sistema de símbolos.

Siguiendo con la presentación de Kaput (1987), hay dos procesos cognitivos que pueden asociarse a los SMS: por un lado, la lectura de la información presentada en un SMS y la codificación de información en cierto SMS; por otro, la elaboración o producción de nueva información una vez que la antigua ha sido codificada. Esta última actividad, a su vez, está dividida en dos: la elaboración sintáctica, en la que el ejecutante se limita a la manipulación de símbolos de acuerdo con las reglas válidas para el caso; y la elaboración semántica, en la que el individuo es capaz de razonar con las entidades representadas por el SMS.

Esta distinción es crucial, porque sirve como marco para analizar en términos semánticos el problema de la construcción del sentido. En efecto, la sola competencia para ejecutar algoritmos en forma estrictamente sintáctica, que en muchos casos es el indicador utilizado para evaluar los desempeños en Matemática, no puede ser considerada como suficiente evidencia de haber conseguido construir significados; en efecto, una persona puede llevar a cabo rutinas de cálculo tipificadas, y no ser capaz de adecuar estas rutinas a casos similares, o no poder anticipar resultados basándose en argumentos sobre el campo de referencia, o no lograr interpretar los resultados obtenidos en términos del mundo representado.

Para Duval (1998, p. 175) "las representaciones semióticas son producciones constituidas por el empleo de signos que pertenecen a un sistema de representación, el cual tiene sus propios constreñimientos de significancia y funcionamiento”. Denomina sémiosis al proceso de construcción de una representación semiótica, en tanto noésis es la construcción conceptual de un objeto, y señala que ambas son inseparables, en tanto no es posible la noésis sin la sémiosis.

Aunque Duval (1998) no hace mención a los SMS como tales, señala características de los sistemas semióticos que permiten ver la proximidad de su planteo con el de Kaput (1987). Indica que para que un sistema semiótico sea un registro de representación debe permitir realizar tres operaciones: la formación de una representación identificable (texto, gráfica, expresión algebraica, entre otros), el tratamiento (transformación de una expresión en otra dentro del mismo registro) y la conversión (transformación de una expresión en un registro, en otra, en un registro diferente). 
En la posición de Duval (1998) debe reconocerse, como señala Kaleff (2007), la asunción de que las entidades matemáticas son de carácter abstracto, y por lo tanto, el acceso a ellas sólo se puede hacer a través de sus representaciones. Más aún, sólo la disponibilidad simultánea de representaciones en diferentes registros semióticos y la capacidad de articularlos a través de las operaciones referidas antes, permite la adecuada conceptualización.

Comparando la posición de Duval (1998) con la de Kaput (1987), la operación de tratamiento presenta una analogía a las transformaciones sintácticas, en tanto la conversión resulta similar a la actividad semántica. Esta asociación puede verse desde otra perspectiva al notar que, para ambos, la adquisición de un concepto requiere la posibilidad de manejar deferentes representaciones o registros.

Para Kaput (1987) sintaxis y semántica son nociones simétricas, en el sentido de que lo que en un sistema de representación puede considerarse sintáctico, al revisarse desde otra representación es semántico. Por ejemplo, consideremos una función real de variable real; por un lado, podemos representarla por medio de su fórmula algebraica y por otro, a través de su gráfica cartesiana; una transformación sintáctica en la representación algebraica consistente en la construcción de una nueva función g dada por $g(x)=f(x-a)$ tiene una interpretación semántica en la representación gráfica consistente en que la gráfica de g se obtiene de la de $\mathrm{f}$ por medio de una traslación. Pero si nuestro punto de partida es la representación gráfica, la operación sintáctica de trasladar una gráfica se interpreta semánticamente en la representación algebraica con la ecuación $g(x)=f(x-a)$.

Este mismo asunto, mirado desde la perspectiva de Duval (1998), se interpreta como la posibilidad de que un tratamiento en un registro (cambio en la ecuación algebraica) puede asociarse a un tratamiento en otro registro (traslación de una gráfica) a través de un proceso de conversión en el que se reconoce que la misma entidad (la función $f$ ) está representada simultáneamente en dos registros y algunos tratamientos en uno pueden tener un correlato en el otro. Es de destacar, como señala Guzmán (1998, p. 6) que "Este traslado da lugar a fenómenos de congruencia y no congruencia semántica”, es decir, en algunos casos es posible encontrar tratamientos en cada registro que se corresponden, en tanto en otros esta correspondencia, si existe, es difícil de establecer.

En otro orden, Duval (1998) destaca que las dificultades para aprender el pensamiento matemático frecuentemente están ligadas a que se pretende enseñar matemáticas como si la sémiosis fuera una operación de inferior categoría que la noésis. 
Esto conduce a la constatación de que los SMS no suelen ser objeto de enseñanza explícita. Cuando se enseñan, frecuentemente se lo hace de manera independiente de los problemas que causaron su origen o de una reflexión sobre sus usos y ventajas, y existe evidencia de que su uso no se aprende como efecto colateral de la ejecución de tareas matemáticas tal como informa Vega (1995).

En relación con las dificultades en la adquisición de los SMS que se mencionaron antes, Rubinstein y Thompson (2001) presentan una serie de desafíos a los que se enfrentan los aprendices en su toma de contacto con los SMS: al tratar de verbalizar información presentada en un formato simbólico, resulta que puede ser necesario utilizar un discurso bastante extenso y difícil de articular correctamente; por otro lado, la misma formulación simbólica puede ser verbalizada de diferentes maneras, todas ellas equivalentes desde el punto de vista estrictamente matemático, pero que marcan matices de diferencia en el momento de planificar o ejecutar acciones; en ocasiones, las formulaciones simbólicas no se leen de derecha a izquierda, y los procesos de búsqueda a derecha e izquierda o hacia arriba y abajo, para obtener una lectura correcta, pueden ser engorrosos.

Frente a estas dificultades debe señalarse un aspecto que Romberg (1991) resalta:

[...] el poder de la Matemática reside realmente en que un pequeño número de símbolos y de afirmaciones simbólicas pueden ser utilizadas para representar un conjunto amplio de situaciones problema distintas. La identificación y la utilización de los símbolos puede organizarse en ámbitos como los enunciados simbólicos que caracterizan el ámbito, las tareas implicadas que deben llevarse a cabo, las reglas que deben seguirse para representar, transformar y realizar los procedimientos y el conjunto de situaciones que generalmente se han utilizado para crear los símbolos, las relaciones entre los mismos y las reglas significativas (ROMBERG, 1991, p. $374)$.

En el desarrollo de tareas matemáticas de cierta complejidad, como la formulación de modelos, la construcción de generalizaciones a partir del reconocimiento de patrones, la deducción de enunciados a partir de otros tomados como premisas, entre otras, los SMS desempeñan el rol de representar las entidades matemáticas involucradas en la tarea, y las relaciones que se establecen entre ellas.

Las reglas sintácticas de construcción y de transformación de expresiones simbólicas están frecuentemente inspiradas en las propiedades de esas entidades, pero, en otros casos, estas reglas son las que proporcionan nuevas formas de interpretación semántica.

Un debate que se suma a este escenario es el que se plantea entre las siguientes posiciones extremas: por un lado, la que establece que un desempeño exitoso en la ejecución de algoritmos es suficiente evidencia de un aprendizaje significativo en Matemática; por otro, 
la que afirma que las habilidades de cálculo están, hasta cierto punto, separadas del conocimiento conceptual.

Al referirse a esta discusión, Berger (2004) asume una postura basada en las nociones de Vygotski $(1986,1994)$ para definir lo que llama uso funcional de los signos. Entiende por signo no simplemente un símbolo, sino un conjunto de ellos, como pueden ser una gráfica, una definición o un algoritmo. Sostiene que los aprendices usan nuevos signos con dos finalidades: una, comunicativa, en la que el estudiante usa el signo para interactuar con otros (pares, profesores) incluso aunque su comprensión del signo sea incompleta o inmadura; otra, conceptualizadora, en la que el signo sirve como un elemento con el que organiza sus ideas matemáticas, mediando, además, en un proceso de socialización al compartir significados del signo con otros actores, incluso con la comunidad matemática.

En este sentido, afirma que el uso funcional de los signos, a la vez de ser condición necesaria para la construcción de significados matemáticos, se constituye en productor de al menos parte de esos significados.

Este uso funcional de los signos se da a través de diferentes clases de actividades, entre las que se cuentan, entre otras, ejecución rutinaria de algoritmos, aplicaciones (tanto internas en la disciplina como en la construcción de modelos fuera de ella), procesos de resolución de problemas, reconocimiento y representación de patrones.

Esta misma línea es asumida por Radford (2000) al estudiar la forma en que estudiantes principiantes usan símbolos y los dotan de significados al realizar tareas de generalización de patrones. Declara que su posición teórica está basada en dos ideas principales: la primera, siguiendo a Vigotsky, es que el funcionamiento cognitivo no sólo está ligado con el uso de los signos sino que, además, es afectado por este uso; la segunda es que los signos que un individuo usa forman parte de un sistema cultural simbólico trascendente al individuo.

Estas dos bases teóricas permiten estudiar el problema de la adquisición de los SMS desde una nueva perspectiva, no tanto desde la pregunta ¿qué representan los signos? sino ¿qué permiten hacer?, es decir, de qué manera los SMS se constituyen en herramientas cognitivas para la construcción del conocimiento matemático, y esto último en el marco de los contextos socioculturales en los que se da la actividad.

Lo expuesto hasta aquí explica por qué un elemento importante en los aprendizajes matemáticos se relaciona con la adquisición de pericia en el uso de los SMS. Por ello es pertinente la interrogante acerca de si, en el marco de un curso de Matemática, es efectiva la 
enseñanza del rol de los SMS para promover aprendizajes que habiliten un uso experto de los SMS por parte de los estudiantes.

\section{Restricciones para el diseño de la experiencia}

Esta experiencia fue diseñada con la intención de que se llevara a cabo sin alterar las estructuras de enseñanza habituales en la institución donde se implementó, de forma que pudiera transformarse, en caso de resultar exitosa, en una práctica docente corriente. Esta decisión impuso algunas restricciones al diseño:

a) Imposibilidad de seleccionar un mismo profesor para los dos grupos de Álgebra Lineal y otro para los dos grupos de Cálculo.

b) Necesidad de usar las instancias de evaluación de los cursos para proponer las pruebas (cuestionarios preliminar y final en Álgebra Lineal, tareas de transferencia en Cálculo)

c) Separación administrativa de los sujetos en dos grupos naturales, de los que se pudo elegir por sorteo un grupo como experimental y el otro como de control.

Los profesores a cargo de los cursos, que aceptaron participar de la experiencia, tuvieron una reunión previa con el investigador, en la que se explicó la finalidad de la experiencia y se acordaron detalles como los plazos de entrega y devolución de las tareas. Se solicitó a los profesores que no introdujeran cambios en sus prácticas motivadas por el hecho de ser parte de una investigación.

\section{Instrumentación de la experiencia}

\subsection{Hipótesis}

Como resultado de la intervención didáctica en el curso de Álgebra Lineal, se esperaba que hubiera diferencias significativas en los desempeños de los grupos experimental y de control en el post-test. También se esperaba poder explicar estas diferencias a partir de la clasificación de los estudiantes, según el resultado del pre-test, en dos categorías, rendimiento bajo, rendimiento alto.

En relación con la posibilidad de transferencia, se esperaba que en las tareas propuestas a los estudiantes del curso de Cálculo, los que formaban parte del grupo 
experimental de la intervención en el curso de Álgebra Lineal tuvieran mayor calificación que el del resto.

\subsection{Diseño experimental}

\subsubsection{Sujetos}

32 alumnos ingresantes en el año 2010 a carreras de la FIT $^{1}$.

Estos alumnos se distribuyen en dos grupos para la asignatura Álgebra Lineal. Mediante sorteo se seleccionó uno de los grupos de Álgebra Lineal como experimental (designado de aquí en adelante como GE, grupo al que se aplicó la intervención) y el otro como de control (que denota en lo sucesivo con GC, que no recibió la intervención). Estos grupos quedaron constituidos al final del curso por 12 y 20 estudiantes, respectivamente.

De los alumnos anteriores, 27 participaron del curso de Cálculo, 10 de GE y 17 de GC.

El número inicial de alumnos en cada grupo resulta de la organización administrativa, que determina grupos con números similares de estudiantes, lo que luego se ve afectado por el abandono que se registra en el transcurso de la enseñanza.

\subsubsection{Intervención, instrumentos y materiales}

En el caso del curso de Álgebra lineal, se diseñó una intervención didáctica con la intención de enseñar aspectos del uso de los SMS. En un estudio anterior de Lacués (2010) se habían puesto a prueba dos intervenciones didácticas para enseñar el uso de los SMS en cursos de Álgebra Lineal. Se optó por la que se describe a continuación.

Como parte del sistema de evaluación del curso, cada estudiante debe resolver en clase un conjunto de tareas, cada una de las cuales se refiere a los temas tratados durante la semana en curso. Estas tareas son corregidas, en general, por el profesor de cada grupo y se da a cada alumno la calificación obtenida en el plazo de una semana.

Para esta instancia se seleccionaron ejercicios que tuvieran como peculiaridad ser especialmente apropiados para ejemplificar alguno de los usos de los SMS.

\footnotetext{
${ }^{1}$ Facultad de Ingeniería y Tecnologías de la Universidad Católica del Uruguay (UCU).
} 
Además, el profesor encargado de la corrección de las tareas fue el mismo, diferente de los profesores a cargo de los grupos. Esta decisión se tomó con la finalidad de minimizar la posibilidad de existencia de diferencias de criterios de corrección.

Los integrantes del GC recibieron sólo la calificación de la tarea, mientras que los del GE, además, fueron instruidos a través de comentarios del profesor que corrigió las tareas, indicando aciertos o errores cometidos en el uso de los SMS al resolver la tarea, o enfatizando de qué forma los SMS contribuyeron a la construcción y comunicación de la solución. A los estudiantes de GE, además, se les permitió hacer consultas adicionales al profesor corrector sobre los comentarios realizados.

Para estudiar los efectos de esta intervención se utilizó un procedimiento de pre-post test.

El cuestionario usado para el test inicial consistió en diez preguntas de múltiple opción, con cuatro posibles respuestas y una sola correcta, clasificadas según el aspecto del uso de los SMS que fuera necesario para su resolución, de acuerdo con las siguientes categorías: traducción, traducción y tratamiento, reconocimiento.

Este cuestionario se incluyó en el diagnóstico al ingreso, entre ítems que indagaban sobre otras cuestiones.

Para confeccionar el post-test se agregaron cinco ítems al pre-test, referidos a temas tratados en el curso de Álgebra Lineal. El criterio para clasificar estos nuevos ítems fue el mismo que el del pre-test. Este cuestionario se planteó a los estudiantes en la misma jornada de la evaluación final del curso, una vez finalizada ésta. Por este motivo, esta instancia resultó ser muy larga y agotadora para la mayoría de los estudiantes y fue una concesión obligada por las restricciones al diseño enumeradas antes.

Para estudiar la transferencia de los aprendizajes obtenidos acerca del uso de SMS en Álgebra Lineal, se diseñaron dos tareas sobre aspectos del uso de SMS que habían sido tratados en la intervención didáctica en este curso, pero sobre contenidos tratados en el curso de Cálculo.

Estas tareas fueron propuestas a los estudiantes en una forma que ya había sido ensayada en otros momentos del curso, para evitar que fuera una situación novedosa para ellos. Consistió en dedicar la primera mitad de una clase a la resolución individual de las dos tareas, en tanto en la segunda parte se pidió a los estudiantes que, reunidos libremente en grupos de tres o cuatro, revisaran lo que habían hecho y escribieran un breve informe 
comentando lo que la discusión grupal había aportado a su comprensión de las soluciones de las tareas.

Luego se compararon los desempeños en la resolución de los ejercicios de los grupos formados, por un lado, por los estudiantes del GE que también cursaban Cálculo, y por otro el de los estudiantes de GC que también lo hacían.

\subsubsection{Análisis de los resultados}

Teniendo en cuenta el número de alumnos que integraba cada grupo, se reconoció, desde el inicio, que existía una gran incertidumbre en los resultados que cualquier test pudiera arrojar.

Para efectuar el análisis de los resultados de los testes inicial y final se definieron diferentes variables, que se detallan a continuación:

a) Pretest indicó el porcentaje de respuestas correctas de cada estudiante en el test inicial.

b) Postest indicó el porcentaje de respuestas correctas de cada estudiante en el test final.

c) Dif se definió como el porcentaje de las diferencias entre el número de respuestas correctas de cada estudiante en los testes final e inicial en los ítems comunes a ambos cuestionarios.

d) Difcom es la diferencia entre Postest y Pretest.

Todos los cálculos que se refieren en lo sucesivo fueron realizados con SPSS V15. La Tabla 1 muestra las medias de las variables definidas.

Tabla 1 - Media de las variables Pretest, Postest, Dif y Difcom

\begin{tabular}{ccc}
\hline & GE & GC \\
\hline Pretest & 0,642 & 0,735 \\
Postest & 0,717 & 0,700 \\
Dif & 0,067 & $-0,055$ \\
Difcom & 0,064 & $-0,072$ \\
\hline
\end{tabular}

Fuente: Desarrollado por el autor

La variable Pretest fue usada en dos direcciones diferentes:

a) por un lado, para establecer si existían diferencias significativas entre GE y GC al comienzo del curso;

b) por otro, para clasificar a los estudiantes en dos grupos de rendimiento: bajo (hasta seis respuestas correctas, es decir, Pretest menor o igual que 0,6), alto (más de seis respuestas 
correctas, o sea, Pretest mayor que 0,6); esta clasificación fue hecha en preparación de análisis posteriores, como se comentará más adelante.

Los resultados de Pretest mostraron que no existían diferencias significativas entre los grupos GE y GC. Tampoco se constataron diferencias significativas entre los respectivos subgrupos de rendimiento alto, por un lado, o de rendimiento bajo, por otro. Para este estudio se usó una prueba de Mann-Whitney.

Esto permitió considerar a los grupos y sus respectivos subgrupos como equivalentes al comienzo de la intervención, por lo que eventuales diferencias finales podrían atribuirse a ella.

Por un lado, interesaba estudiar la evolución de cada grupo. Una primera constatación a partir de la Tabla 1 es que GE aumentó su desempeño en el test final respecto al inicial, no sólo en las preguntas comunes a ambos cuestionarios, sino también al considerar el cuestionario final completo, en tanto GC lo disminuyó.

En segunda instancia, se compararon (a través de la prueba de Mann-Whitney) las diferencias entre los desempeños de cada grupo en el post- test respecto al pre-test utilizando la variable Difcom. Concretamente, se encuentran diferencias significativas entre GE y GC en esta variable Difcom. La Tabla 2, dada a continuación, resume estos resultados presentando la significación de las diferencias entre las variables en relación con los grupos o subgrupos mencionados. En ella se aprecia que la diferencia significativa entre ambos grupos puede asociarse a una diferencia en los rendimientos de los grupos altos, mientras que en los grupos bajos esto no ocurre.

Se constató una evidencia en el mismo sentido con la variable Dif, que si bien no muestra diferencias significativas entre GE y GC ni entre sus subgrupos bajos, sí la registra en sus grupos altos.

Tabla 2 - Niveles de significación para las diferencias en la variable Difcom en relación con los grupos GE y GC y sus respectivos grupos de rendimiento alto

\begin{tabular}{cc}
\hline GE-GC & Difcom \\
GE-GC altos & 0,049 \\
GE-GC bajos & 0,019 \\
\hline
\end{tabular}

Fuente: Desarrollado por el autor

Para el estudio de la transferencia a otros contextos de los aprendizajes conseguidos en Álgebra Lineal, se consideraron los desempeños de los estudiantes en dos tareas propuestas en el curso de Cálculo. Éstas trataban sobre algunos de los aspectos sobre cuya enseñanza se hizo énfasis, concretamente en trabajos de tratamiento (Tarea 1) o en la representación de generalizaciones (Tarea 2). 
En la Tabla 3 se dan las medias y los niveles de significación de las diferencias de desempeño constatadas entre GE y GC.

En ellas se puede apreciar que, en ambas tareas, el desempeño de los integrantes de GE fue significativamente superior al de los de GC. Para establecer este resultado se utilizó un Student's t-test, considerando los resultados de cada grupo en las tareas como muestras independientes.

Tabla 3 - Medias de las tareas de Cálculo y nivel de significación de las diferencias en

\begin{tabular}{ccc} 
& relación con GE y GC & \\
\hline & Tarea 1 & Tarea 2 \\
Media de GE & 1 & 0,75 \\
Media de GC & 0,65 & 0,47 \\
Significación & 0,017 & 0,008 \\
\hline
\end{tabular}

Fuente: Desarrollado por el autor

\section{Análisis didáctico de las tareas}

Cada una de las tareas propuestas a los estudiantes fue analizada por profesores de Matemática, que establecieron qué aspectos del uso de los SMS eran relevantes para obtener la solución.

Por otro lado, luego de corregidas las tareas se construyó, para cada una, un índice de los niveles de desempeño detectados, como forma de categorizar las soluciones y asignarles una calificación. Para cada estudiante se llevó un registro longitudinal de las calificaciones obtenidas, con la intención de registrar evidencias acerca de su evolución.

A continuación se presentan tres de las tareas, junto con el análisis de los expertos y los niveles de desempeño. También se reseñan las recomendaciones que se hicieron a cada estudiante según el desempeño que hubiera mostrado.

\subsection{Tarea 1}

Suponga que se define una operación * en el conjunto $\boldsymbol{R}$ de los números reales por medio de $x^{*} y=x+x . y-y$, donde la suma, el producto y la resta son las habituales en $\boldsymbol{R}$.

a) Pruebe que 0 es neutro a la derecha de esta operación, o sea, $x * 0=x$ cualquiera sea $x$.

b) Averigüe si la propiedad conmutativa es válida para *, es decir, si $x^{*} y=y^{*} x$ para cualesquiera $x$ e $y$.

c) Halle, si existen, tres números reales $a$, $b$ y c tales que $(a * b)^{*} c=a^{*}\left(b^{*} c\right)$.

d) Halle una condición necesaria y suficiente para $x^{*} y=y^{*} x$. 
Esta tarea plantea preguntas acerca de una ley de composición definida arbitrariamente, en términos de las operaciones usuales en el conjunto de los números reales.

Para resolverla, debe tenerse en cuenta dos conjuntos de reglas sintácticas, el de las operaciones habituales en los reales, por un lado, y por otro, la regla provista por la definición. Por lo tanto, en este problema se plantean solamente procesos de transformación en el registro algebraico, y no se requieren contenidos propios del curso de Álgebra Lineal, por lo que fue de las primeras tareas propuestas.

En este problema se identificaron los siguientes seis niveles de desempeño:

0 - No consigue aplicar la definición.

1 - Presenta errores de cálculo.

2 - No extrae conclusiones de las ecuaciones que plantea, o las extrae en forma incompleta.

3 - Cree que es suficiente considerar la forma para decidir si dos expresiones son diferentes; presenta errores procedimentales.

4 - Cree que es suficiente considerar la forma para decidir si dos expresiones son diferentes; opera según procedimientos.

5 - Opera según procedimientos y obtiene las conclusiones requeridas.

A los estudiantes del nivel 0 se les sugirió que comenzaran por tomar algunos pares de reales para efectuar con ellos la operación *, y explorar con esos ejemplos los enunciados de las partes a) y b).

A los que consiguieron el nivel 1 se los orientó para corregir sus errores y para que vieran cómo éstos afectaban sus posibilidades de completar la tarea.

A los que estuvieron en el nivel 2 se les interrogó específicamente sobre cuáles eran las conclusiones que podían extraer de los resultados que habían obtenido.

Tanto a los del nivel 3 como a los del 4 se les confrontó con ejemplos donde la forma algebraica de dos expresiones era diferente, pero ambas eran equivalentes. A los del 3, adicionalmente se les indicó cómo superar los errores cometidos.

A los del nivel 5 se les comentó que su solución alcanzaba un grado de calidad alto, y, en algún caso, se sugirió explorar vías de solución diferentes de las que habían conseguido.

Los estudiantes que consiguieron hasta el nivel 2 parecen tener escasas competencias en el uso de los SMS, lo que hace que la enseñanza que se les planteó esté orientada a aspectos más básicos; entretanto los de los niveles a partir del 3 muestran un desarrollo que permite suponer que podrán aprovecharse de sugerencias más sutiles, como la de explorar soluciones alternativas o construir la noción de equivalencia entre expresiones. 
Una constatación que pudo hacerse al considerar la sucesión de trabajos es que, en general, los estudiantes que tuvieron un desempeño bajo en estas primeras tareas, no lo superaron demasiado a lo largo del curso, y consiguieron, en general, un promedio de calificación inferior al 0,5; en cambio, los que tuvieron rendimiento alto, consiguieron luego mantenerlo o superarlo, para obtener finalmente promedios superiores a 0,5 .

Para la tarea que sigue se necesitan algunos de los contenidos que se enseñan en el curso de Álgebra Lineal (concretamente sobre álgebra matricial), por lo que fue propuesta promediando el semestre. Lo que se busca con esta tarea es estudiar el vínculo entre el uso de SMS y la capacidad para detectar un patrón y representarlo en forma general.

\subsection{Tarea 2}

a) Sea $\left(\begin{array}{l}\mathrm{a} \\ \mathrm{b}\end{array}\right)$ una matriz $2 x 1$. Encuentre una matriz cuadrada, A, de orden 2 tal que $A\left(\begin{array}{l}a \\ b\end{array}\right)=\left(\begin{array}{l}b \\ a\end{array}\right)$.

b) Tome ahora $\left(\begin{array}{l}\mathrm{a} \\ \mathrm{b} \\ \mathrm{c}\end{array}\right)$, una matriz 3x1. Encuentre una matriz cuadrada, B, de orden 3 tal que $B\left(\begin{array}{l}a \\ b \\ c\end{array}\right)=\left(\begin{array}{l}c \\ b \\ a\end{array}\right)$.

c) Plantee la situación análoga a las de las partes a) y b) para una matriz $4 x 1$.

d) Generalice las situaciones anteriores a una matriz $n x 1$, con $n$ cualquiera. Explique los motivos de la elección de la notación que utilice, y trate de justificar en términos simbólicos los procesos que realice.

Esta tarea propone la construcción de una generalización a partir de casos particulares, a través de la generación de una representación simbólica adecuada (introducción de subíndices); el trabajo se realiza dentro del registro algebraico y podría considerarse que consiste en la elaboración de una regla sintáctica, concretamente la que muestra que el producto de una cierta matriz por un vector tiene por resultado invertir el orden en las coordenadas del vector. 
La resolución de las tres primeras partes consiste en la construcción de una matriz. Si bien existe un procedimiento algorítmico para dar la respuesta, es bastante trabajoso aplicarlo, por lo que se espera que la respuesta sea dada apelando a algún procedimiento heurístico. La detección del patrón que resulta al observar los resultados conseguidos no parece presentar mayores dificultades. La generalización implica la introducción de un subíndice para representar un número desconocido de entradas en la matriz nx1, y en elaborar una regla sintáctica por la cual se indica cuál es el resultado del producto con la matriz que se pide hallar. Una vez hecho esto, para describir esta matriz se deberá usar un par de subíndices, indicando cuánto vale la entrada en la matriz correspondiente a cada par.

Los niveles de desempeño registrados fueron los siguientes:

0 - No consigue formular el problema.

1- No hay indicios de dónde obtiene el resultado; da notaciones simbólicas no adecuadas.

2 - Plantea y resuelve un sistema en las dos primeras partes, pero ni induce un resultado a partir de allí ni generaliza el planteo.

3 - No hay indicios de dónde obtiene el resultado de las dos primeras partes; induce una generalización y usa una notación adecuada.

4 - Plantea y resuelve un sistema en las dos primeras partes y luego induce el resultado de las restantes; no introduce subíndices.

5 - Plantea y resuelve un sistema en las dos primeras partes y luego induce el resultado de las restantes; introduce subíndices; o plantea y resuelve un sistema para cada parte; usa notación adecuada.

A los estudiantes del nivel 0 se les detalló la solución de las partes a) y b) y se les mostró cómo podría conjeturarse cuál sería la de la parte c).

Los que integraban el nivel 1 fueron corregidos en las notaciones, explicando cómo adecuarlas, y se les sugirió que reescribieran la solución conseguida, explicitando cómo la habían conseguido y teniendo en cuenta las sugerencias sobre la notación.

A los que consiguieron el nivel 2 se les planteó la solución de la parte c) y se les dieron pautas para reconocer el patrón y escribir la generalización, en particular señalando la conveniencia de usar subíndices.

Los que lograron el nivel 3 fueron aconsejados para que expusieran cómo había resuelto las primeras partes.

A quienes llegaron al nivel 4 se le indicó cómo introducir los subíndices para representar la generalización. 
A los del nivel 5 se les sugirió que exploraran el camino alternativo al que habían recorrido: si habían inducido la solución, que intentaran obtenerla formalmente a partir de la construcción de un sistema; si, en cambio, habían trabajado en base a la construcción de los sistemas, se les pidió que se preguntaran cómo podían haber inducido el resultado.

Al igual que en la Tarea 1, podrían clasificarse los estudiantes en los que obtuvieron hasta el nivel 2 y en los que obtuvieron del 3 en adelante, y las consideraciones anteriores son pertinentes en este caso.

La siguiente tarea es de las últimas propuestas, debido a los contenidos matemáticos que requiere (noción de independencia lineal) que son tratados sobre el final del curso.

\subsection{Tarea 3}

Suponga que el subconjunto $\{u, v\}$ del espacio vectorial $V$ es LI. Para probar que el subconjunto $\{u, u+v\}$ es LI se puede proceder asi:

$\{u, u+v\}$ es $L I \Leftrightarrow(\alpha u+\beta(u+v)=o \Rightarrow \alpha=\beta=0) \Leftrightarrow((\alpha+\beta) u+\beta v)=o \Rightarrow \alpha=\beta=0)$

Pero como $\{u, v\}$ es LI, resulta que $(\alpha+\beta) u+\beta v)=o \Rightarrow \alpha+\beta=\beta=0$ y la única solución del sistema

$$
\left.\begin{array}{rl}
\alpha+\beta & 0 \\
\beta & =0
\end{array}\right\} \text { es } \alpha=\beta=0 \text { con lo que se prueba lo deseado. }
$$

a) El problema análogo para tres vectores consiste en probar que si $\{u, v, z\}$ es LI entonces $\{u, u+v, u+v+z\}$ es LI. Adapte la solución anterior a este caso.

b) Formule y resuelva el problema correspondiente a cuatro vectores.

c) Analice los problemas anteriores y trate de detectar patrones en las soluciones que haya obtenido. Generalice la formulación y el proceso de solución del problema para una cantidad cualquiera de vectores. Explique la notación que elija para representar el problema, y sea tan detallado como crea necesario para establecer su argumento.

Para esta tarea no es posible mantenerse en el registro algebraico, sino que deben llevarse a cabo procesos de conversión, concretamente en la aplicación de la definición de independencia lineal.

El conocimiento del contenido matemático es aquí crucial para obtener la solución, lo que puede hacer más difícil reconocer el patrón para llevar adelante la generalización.

En este momento es importante hacer un comentario. El uso de los SMS no puede desvincularse del contenido matemático en el que se aplica. Es más, retomando a Duval (1998), la construcción de adecuadas representaciones está asociada con el propio proceso de 
adquisición del concepto. Por esto, aunque el rol de los SMS pueda, en algún aspecto, ser similar en dos tareas diferentes (por ejemplo, construir una generalización), estos pueden tener que funcionar en diferente forma para conseguir la solución.

Los niveles de desempeño registrados fueron los siguientes:

0. Plantean las partes a) y b) pero no generalizan.

1. Plantea una generalización inadecuada.

2. Plantea una adecuada notación para la generalización, pero no termina de formularla.

3. Generaliza usando una notación inadecuada, sin subíndices o con subíndices para los vectores pero no para los escalares.

4. Generaliza adecuadamente.

Al igual que en los casos anteriormente descritos, existe una marcada diferencia en el uso de los SMS entre quienes alcanzan los niveles hasta el 2 y los otros. Las sugerencias y comentarios efectuados a los estudiantes en este caso fueron similares a las anteriores, aunque se insistió con los de calificación más baja en la necesidad de revisar los contenidos matemáticos involucrados en la tarea.

Por otro lado, aunque las tareas 2 y 3 consisten en generalizaciones, la 3 registró un rendimiento menor que la 2. Esto puede tener varias explicaciones (por ejemplo, en el momento de la entrega de la tarea 3 los estudiantes estaban rindiendo las pruebas finales y eso debe haber afectado su dedicación), pero también es posible que el contenido matemático (álgebra matricial en una, independencia lineal en la otra) haya sido en parte la razón de esta disminución de rendimiento.

\section{Conclusiones}

La inexistencia de diferencias en el pre-test entre GE y GC permite asociar las diferencias detectadas a la intervención didáctica desarrollada.

Un primer indicador del éxito de la intervención es el resultado que marcó calificaciones más altas, aunque no significativamente, entre los desempeños de GE en los testes inicial y final, en tanto GC presentó un leve desmejoramiento.

La existencia de diferencias significativas al comparar cuánto cambian GE y GC con la variable Difcom, es un resultado fuerte a favor de la intervención, sobre todo cuando se lo considera en conjunto con el anterior. Significa que en conjunto el grupo que recibe enseñanza intencional cambia más, y en sentido positivo, que el que sólo recibe la enseñanza tradicional. 
Estos hechos son consistentes con la crítica de Duval (1998) y los resultados de Vega (1995), marcando que en ausencia de enseñanza intencional, no es razonable esperar que se produzcan aprendizajes en el uso de los SMS simplemente por exposición a tareas matemáticas donde se utilicen.

Por otro lado, la existencia de estas diferencias puede justificarse a partir de los grupos de rendimiento alto, que son significativamente distintos en las variables Dif y Difcom.

Un argumento explicativo puede elaborarse a partir de la noción de Zona de Desarrollo Proximal de Vigotsky. En efecto, los estudiantes de desempeño alto pueden entender e incorporar a sus prácticas las sugerencias y comentarios del profesor. Dicho de otra manera, la enseñanza que propone esta intervención está situada de manera que permite la adquisición de las habilidades en el uso de los SMS que están siendo enseñadas. En otro sentido, los de desempeño bajo muestran un grado insuficiente de apropiación de las habilidades de uso de los SMS, como para poder aprovecharse de la enseñanza impartida, lo que plantea la necesidad de modificar el tipo de intervención para aproximar la enseñanza a sus modos de aprendizajes actuales.

Desde el punto de vista educativo, este resultado es importante porque implica al menos dos consecuencias:

a) es posible desarrollar diagnósticos al ingreso a la universidad que permitan anticipar cuáles estudiantes podrán sacar provecho de la enseñanza usual en el ámbito universitario y cuáles difícilmente lo consigan;

b) para estos últimos deben diseñarse mallas curriculares y, sobre todo, formas de enseñanza alternativas que promuevan sus aprendizajes, y, en particular, la apropiación de habilidades en el uso de SMS, teniendo efectivamente en cuenta su punto de partida y sus estilos de construcción de conocimientos.

Este punto parece de la mayor importancia. Si se aceptan perspectivas como la de Radford (2000) que afirman que el uso de los signos es, a la vez que un medio para el quehacer matemático, un elemento modificador del funcionamiento cognitivo del individuo, así como que son una forma de comunicar y socializar el conocimiento como sostiene Kaput (1987), y además contribuyen a la construcción de los conceptos matemáticos, tal como afirma Duval (1998), entonces capacidades no desarrolladas para aprehender las habilidades que permiten el uso de los SMS son determinantes en la consecución de aprendizajes matemáticos significativos. 
Por eso, la elaboración de alternativas didácticas que pongan estos aprendizajes al alcance de los estudiantes que a su ingreso a la universidad muestran una escasa habilidad, se convierte en una prioridad educativa.

El resultado de la tarea de transferencia es importante, porque proporciona evidencia en el sentido de que la instrucción intencional en el uso de SMS promueve aprendizajes que pueden ser usados en otros contextos. Los trabajos de tratamiento dentro de un registro están presentes en cualquier actividad matemática, lo mismo que el reconocimiento de patrones y su representación simbólica como una generalización.

Por esta razón, desde el punto de vista de la educación matemática, este resultado permite plantear la cuestión de si es o no relevante sobre qué contenidos matemáticos se enseñe acerca del uso de los SMS, o si lo sustancial es que esa enseñanza se efectúe.

Preguntas que se pueden formular a partir de este resultado son: ¿aprendizajes conseguidos en cualquier área de contenidos se transfieren a cualquier otra o existe alguna direccionalidad en esta posibilidad de transferencia? ¿es necesario haber conseguido un cierto grado de suficiencia en el uso de SMS para poder llevar a cabo esta transferencia?

A partir del análisis didáctico pueden efectuarse otros comentarios. Aunque los resultados estadísticos no fueron significativos, existe cierta evidencia, al revisar las producciones de los estudiantes, en el sentido de que quienes consiguen tempranamente un nivel desempeño alto, lo mantienen luego pese a la progresiva complejidad de los contenidos matemáticos presentes en las tareas.

En cambio, quienes no consiguen rápidamente un desempeño suficiente, continúan sin conseguirlo, y esto llega a afectar su aprendizaje de los contenidos. Por eso, cabe interrogarse acerca de la forma de organizar la enseñanza para proponer trabajos que permitan a estos estudiantes desarrollar su manejo de los SMS a un nivel de suficiencia. No fue el objeto de este estudio, pero un camino a explorar es el de incluir actividades en grupos conformados intencionalmente con estudiantes de diferentes desempeños en el diagnóstico inicial, como forma de estimular interacciones entre pares que contribuyan a conseguir este desarrollo.

Por otro lado, queda planteada una cuestión en relación con el rol de los SMS en el desarrollo de una tarea, y es el de sus vínculos con los contenidos matemáticos de la tarea. El éxito en conseguir la solución depende, entre otros factores, del reconocimiento de cómo interpretar contextualmente reglas sintácticas para establecer semánticamente relaciones entre las entidades matemáticas en juego. El estudio de estos vínculos no fue una pretensión de este trabajo, pero el análisis de las tareas planteó claramente la importancia de esta interrogante. 


\section{Referencias}

BERGER, M. The functional use of a mathematical sign. Educational Studies in Mathematics, Dordrecht, Holanda, v. 55, p. 81-102, Mar. 2004.

DUVAL, R. Registros de representación semiótica y funcionamiento cognitivo del pensamiento, In: HITT, F. (Ed.). Investigaciones en Matemática Educativa II. México: Grupo Editorial Iberoamérica, 1998. p. 173-201.

GUZMÁN, I. Registros de Representación, el Aprendizaje de Nociones Relativas a Funciones: Voces de los Estudiantes, RELIME, Distrito Federal, México, v. 1, n. 1, p. 5-21, mar. 1998.

KALEFF, A. M. Registros Semióticos e Obstáculos Cognitivos na Resolução de Problemas Introdutórios às Geometrias não-Euclidianas no Âmbito da Formação de Professores de Matemática, Bolema, Río Claro, n. 28, p. 69-94, 2007. Disponible en:

$<$ http://www.periodicos.rc.biblioteca.unesp.br/index.php/bolema/issue/view/735/showToc $>$. Acceso en: 4 abr. 2012.

KAPUT, J. Towards a Theory of Symbol use in Mathematics. En: JANVIER, C. (Ed.). Problems of Representation in the Teaching and Learning of Mathematics. Hillsdale, NJ: Lawrence Erlbaum Associates Publishers, 1987. p. 159-195.

LACUÉS, E. Enseñanza y aprendizaje de los Sistemas Matemáticos de Símbolos, DIDAC, México, n. 56-57, p. 30-36, jul./dic. 2010, ene./jun. 2011. Disponible en: $<$ http://www.uia.mx/web/files/didac/5657.pdf $>$. Acceso el: 30 dic. 2011.

MARTÍ, E.; POZO, I. Más allá de las representaciones mentales, la adquisición de sistemas externos de representación, Infancia y aprendizaje, Madrid, v. 90, p. 11-30, 2000.

PALMER, S. Fundamental aspects of cognitive representation. In: ROSCH, E.; LLOYD, B. B. (Ed.). Cognition and categorization. Hillsdale, NJ: Lawrence Erlbaum Associates, Publishers, 1978. p. 259-303. Disponible en: <http://socrates.berkeley.edu/ plab/pdf/Palmer\%20-\%201978.pdf >. Acceso en: 30 abr. 2012.

RADFORD, L. Signs and meanings in students' emergent algebraic thinking: a semiotic analysis. Educational Studies in Mathematics, Dordrecht, Holanda, v. 42, p. 237-268, mayo 2000.

ROMBERG, T. Características problemáticas del currículo escolar de matemáticas. Revista de Educación, Madrid, v. 294, p. 323-s406, 1991.

RUBINSTEIN, R.; THOMPSON, D. Learning Mathematical Symbolism: Challenges and Instructional Strategies, Mathematics Teacher, Syracuse, U.S., v. 94. n. 4, p. 265-271, abr. 2001. Disponible en: <http://132.68.98.62/Courses/Algebra_206/Algebra\%20-\%202005/Articles/15-MTv94\%20n4\%20265-271-Learning\%20math\%20symbolism.pdf $>$. Acceso en: 30 abr. 2012.

VEGA, E. El uso del lenguaje algebraico en alumnos de Bachillerato. Educación Matemática, México, v. 7, n. 3, p. 27-47, dic. 1995.

VYGOTSKY, L. S. Thought and Language, Cambridge, Mass: MIT Press, 1986.

VYGOTSKY, L. S. The Vygotsky Reader. Oxford: Blackwell Publishers, 1994. 